\title{
Neurobiology of PTSD: A Review of Neuroimaging Findings
}

\section{Sarah N. Garfinkel, PhD; and Israel Liberzon, MD}

$\mathrm{N}$ euroimaging serves as an effective way to investigate the neurocircuitry involved in the development of posttraumatic stress disorder (PTSD). ${ }^{1-3}$ A number of models describing functional neuroanatomy of PTSD symptom development emerged over the past decade, inspired by both basic animal research and an increasing number of human neuroimaging studies. These models have traditionally conceptualized PTSD as a state of heightened responsivity to threat-

Sarah N. Garfinkel, PhD, is with the Department of Psychiatry, University of Michigan, Ann Arbor. Israel Liberzon, MD, is with the Department of Psychiatry, Ann Arbor VA Medical Center, Ann Arbor, Michigan.

Address correspondence to: Israel Liberzon, MD, Department of Psychiatry, Rachel Upjohn Building, 4250 Plymouth Road, Ann Arbor, MI 48109; fax 734-936-7868; or email:liberzon@umich.edu.

Dr. Garfinkel and Dr. Liberzon have disclosed no relevant financial relationships.

doi: 10.3928/00485713-20090527-01 ening stimuli and later as a state of insufficient inhibitory control over-exaggerated threat-sensitivity. They emphasize the centrality of threat-related processing in the pathophysiology of PTSD and therefore account for the "hypersensitivity to threat," which is highly characteristic of PTSD (such as hypervigilance and hyperarousal). Neuroimaging findings in PTSD lend credence to incorporating "hypersensitivity to threat" within the conceptualization of PTSD, and these findings will be discussed within this review.

It is becoming increasingly apparent, however, that the "hypersensitivity to threat" models do not fully capture the full complexity of PTSD, nor the complexity of changes associated with, trauma exposure and PTSD development. For instance, important phenomena associated with PTSD, such avoidance and numbing, generalization of fear, vulnerability, and resilience factors, all need to be further understood in terms of underlying psychological mechanisms and their neurobiological substrates. The dysfunction of neuronal regions in
PTSD, and the implication this has for the disruption of cognitive and psychological processes, will also be discussed.

This review summarizes the neuroimaging findings pertaining to PTSD and PTSD vulnerability and will focus on a few key regions known to be particularly implicated in PTSD pathophysiology, specifically amygdala, nucleus accumbens (ACC), medical prefrontal cortex (mPFC), and hippocampus. It will first start by reviewing key structural alterations associated with PTSD, and then progress to discussing functional abnormalities, with a particular emphasis on symptom provocation studies, cognitive activation studies, and functional connectivity analyses. Finally, neurochemical differences, as revealed via receptor imaging, will be addressed followed by a discussion of potential future directions for PTSD research.

Different neuroimaging modalities, such as single-photon emission tomography (SPECT), positron emission tomography (PET), magnetic resonance spectroscopy (MRS), magnetic resonance 


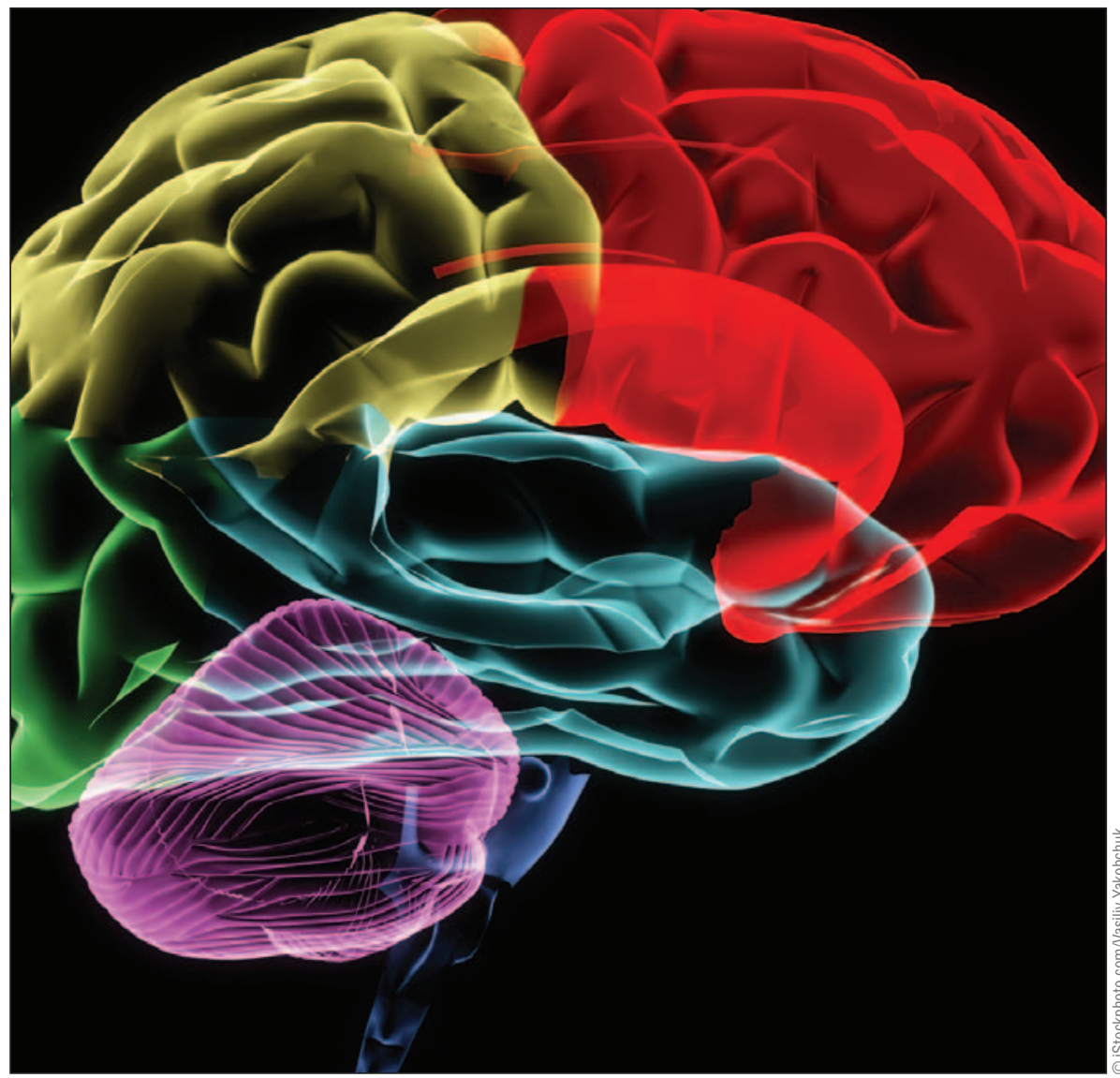

imaging (MRI), and functional MRI (fMRI) have all been used in conjunction with PTSD research. This article will incorporate many of these modalities, with a particular emphasis on MRI in the structural section, and fMRI when dealing with functional studies.

\section{STRUCTURAL ABNORMALITIES ASSOCIATED WITH PTSD}

Structural neuroimaging studies have originally focused on hippocampal volume in PTSD patients, although emerging evidence suggests structural alterations are also present in other regions. The following section will outline key structural alterations associated with PTSD and PTSD vulnerability focusing on the hippocampus, ACC, and amygdala.

\section{Hippocampus}

The hippocampus is a known target for stress hormones, ${ }^{4}$ and abnormalities in hippocampal volumes had been reported in conditions associated with HPA abnormalities like depression and Cushing's disease. ${ }^{5,6}$ Although PTSD has not been associated with hypercortisolemia, studies of structural brain abnormalities in PTSD have originally focused on the hippocampus, which is also known to be involved in explicit/declarative memory, working memory, episodic/autobiographical memory and contextual learning/memory. ${ }^{7,8}$ Individuals with PTSD perform poorly on neuropsychological memory tasks ${ }^{9,10}$ and are impoverished in their autobiographical memories for positive events. ${ }^{11}$ A number of structural MRI studies reported decreased hippocampal volumes in individuals with PTSD. ${ }^{9,12-15}$ MRS studies have reported decreased $\mathrm{N}$-acetylaspartate (NAA) levels in the hippocampus, interpreted as reflecting decreased neuronal integrity. ${ }^{16,17}$ Reductions in hippocampal volumes have ranged from $5 \%$ to $26 \%$, and have tended to be found bilaterally across studies. ${ }^{18}$ It should be noted, however, that a number of studies have not replicated the finding of decreased hippocampal volumes in PTSD. ${ }^{19}$ ${ }^{21}$ These discrepancies suggest that smaller hippocampi may be restricted to subgroups of PTSD, may be secondary to comorbid conditions, or that hippocampal pathology may be subtle and not always detectable using standard morphometric MRI procedures. ${ }^{18}$ Furthermore, it has not been clear whether reported hippocampal changes are acquired signs of PTSD, or potential predisposing factors. Finally results of a recent meta-analysis further support the presence of smaller hippocampal volumes in PTSD patients relative to controls with and without trauma exposure. ${ }^{22}$

A twin study of Vietnam veterans has helped to clarify the question of whether the hippocampal findings reflect predisposing factors or acquired signs of PTSD. Gilbertson et al studied monozygotic twins discordant for trauma exposure, with and without PTSD, and found that both twins with PTSD and their trauma unexposed twin had smaller hippocampi relative to trauma exposed non-PTSD twins and their co-twin. ${ }^{23}$ Moreover, the same group, both PTSD and trauma nonexposed co-twin, showed impaired hippocampus-mediated spatial processing, using a cue configuration task. ${ }^{24}$ These findings offer compelling evidence for reduced hippocampal size serving as a preexisting and potentially vulnerability or predisposing factor for PTSD.

\section{Anterior Cingulate Cortex}

Increasing attention is being devoted to the anterior cingulate cortex (ACC) and the potential role it may play in PTSD. ${ }^{2}$ Significantly, the ACC shares close neuroanatomical relationships with subcortical components of the "central fear system." 25 There is emerging evidence of structural impairment of the ACC associated with PTSD. Manual tracing of the ACC has demonstrated it to be smaller in patients with PTSD, relative to control participants. ${ }^{25}$ Voxel-based morphometry studies have shown reduced grey matter density in the $\mathrm{ACC},{ }^{26}$ and a recent meta-analysis of these studies confirmed the presence of significantly smaller anterior cingulate cortex volume in PTSD patients relative 
to trauma-exposed controls. ${ }^{22}$ Kasai et al performed a VBM analysis in the twin cohort in search of structural abnormalities. They found a significant PTSD diagnosis by exposure interaction in the pregenual ACC, such that combat-exposed PTSD twins were found to have lower gray matter density than their combat-unexposed cotwins and lower than the combat-exposed twins without PTSD and their co-twins. ${ }^{27}$ This supports the inference that pregenual ACC gray matter reduction is likely an acquired sign of PTSD as opposed to a premorbid vulnerability factor. In accordance with these structural findings, there is also evidence to suggest that ACC function in PTSD is impaired, and this will be addressed in the following section.

\section{Amygdala}

There is equivocal evidence to suggest reduced bilateral amygdala volume is associated with PTSD, as studies differ markedly in results obtained. ${ }^{19,28} \mathrm{~A}$ meta-analysis, including data from both adults and children with PTSD from different types of trauma, demonstrated some evidence of smaller left amygdala in PTSD subjects compared with healthy controls, ${ }^{22}$ at the same time highlighting the controversial findings present in the literature. There are a few studies investigating amygdala volume in children with maltreatment-related PTSD, which tend to mirror the inconclusive findings obtained in adults. In one study, no global volume differences between PTSD and control groups were obtained. ${ }^{21}$ In contrast, Carrion et al found a $5.1 \%$ reduction in the volume of the amygdala in children with maltreatment-related PTSD compared with healthy controls, although this result became non-significant when scores were adjusted for total brain gray matter. ${ }^{20} \mathrm{~A}$ recent meta-analyses investigating amygdala volumes in individuals with childhood maltreatment-related PTSD in both child and adult populations did not find PTSD-related reductions in amygdala volume. ${ }^{29}$

\section{Insula}

The insula is highly interconnected with cortical and limbic regions implicated in $\mathrm{PTSD}^{30}$ and is involved in key processes (eg, emotional processing) ${ }^{31}$ that are linked to PTSD pathophysiology; however, to date, only limited evidence suggests the presence of structural abnormalities in the insula in PTSD. One recent study used voxel-based morphometry (VBM) to explore structural differences in gray or white matter volume between subjects with and without PTSD. ${ }^{32}$ The PTSD group had lower gray matter density in insula bilaterally as compared with controls. Differences were also obtained in the left hippocampus and left ACC. These results are in accordance with an earlier whole-brain VBM study that showed PTSD subjects had lower gray matter density in the left insula relative to healthy controls. ${ }^{26}$

\section{FUNCTIONAL STUDIES}

Functional neuroimaging studies have the potential to identify regions involved in the pathophysiology of PTSD, due to the altered function of these regions, like $\mathrm{mPFC}, \mathrm{ACC}$, and amygdala. It is important to note that functional imaging experiments in individuals with PTSD fall within two categories: those which utilize symptom provocation, and those which rely on cognitive activation probes. Studies utilizing symptom provocation to investigate PTSD do so by employing autobiographical stimuli, which are trauma-related (eg, narrative scripts of personal trauma), or alternatively are more general in nature, employing generally evocative but not necessarily autobiographically relevant pictures and sounds. Such studies were the first to emerge, were the first to provide relatively stable and replicable findings, and are still the most common studies in the PTSD functional neuroimaging literature. In contrast, cognitive activation studies assess specific impairments in neuronal processing associated with PTSD via a neurocognitive task (a "probe") that is expected to selectively activate neural circuits implicat- ed in task-related processing. Selectively activating a circuit without eliciting symptoms has a substantial advantage in that this elicits a large number of more general or nonspecific trauma-related responses. Investigators have used cognitive activation strategies to further examine a number of regions implicated in PTSD by symptom provocation studies, such as the $\mathrm{mPFC}$, amygdala, ACC, and hippocampus.

\section{$A C C$ and $\mathrm{mPFC}$}

The ACC is a region that has been activated by many functional neuroimaging studies and has been implicated in different processes involving cognitive-emotion interactions. A variety of evidence supports the existence of functional subdivisions in the ACC, with dorsal ACC supporting cognitive control and error-related processing, while $\mathrm{rACC}$ is involved in the assessment of salience of emotional information and the regulation of emotional responses. ${ }^{33}$ Reduced activation of the ACC in PTSD patients, relative to both control participants with and without trauma exposure, has been repeatedly shown in a variety of symptom provocation studies. Rauch and colleagues used combat-related, emotionally negative, and neutral pictures paired with verbal descriptions (imagery) in combat veterans with and without PTSD. Combat veterans with PTSD had increased $\mathrm{rCBF}$ in ventral anterior cingulated cortex (ACC) and right amygdala when generating mental images of combat-related pictures, but had deceased $\mathrm{rCBF}$ in the $\mathrm{ACC}$ in the combat image viewing versus neutral image viewing contrast. ${ }^{34}$ Another study using combat-related pictures and sounds and PET in 10 combat veterans with and 10 without PTSD revealed decreased blood flow in MPFC (area 25) and other areas in response to traumatic pictures and sounds in PTSD patients. However, non-PTSD control subjects activated the anterior cingulate (area 24) to a greater degree than PTSD patients. ${ }^{35}$ The same group also studied childhood sexual abuse (CSA) subjects (22 women, 10 of whom had PTSD) with 
exposure to traumatic and neutral scripts and PET. The PTSD group showed rCBF increases in posterior cingulate (area 31) and superior and middle frontal gyri bilaterally (Brodmann areas 9 and 10). The PTSD group also showed deactivation in the subcallosal anterior cingulate (area 25) and decreased activation in an adjacent portion of anterior cingulate (area 32). ${ }^{35}$ Using PET and script-driven imagery in 16 subjects with CSA ( 8 with PTSD), Shin and colleagues also reported deactivation of the medial prefrontal and as well as left inferior frontal (Broca's) areas in the PTSD group. ${ }^{36}$ We recently reported the results of a [15O] $\mathrm{H}_{2} \mathrm{O}$ PET, script-driven imagery study of emotionally evocative and neutral autobiographic events in 16 combat veterans with PTSD (PTSD patients [PP], 15 combat veterans without PTSD (combat controls [CC]), and 14 healthy, age-matched, control subjects (noncombat controls [NC]) that allow to isolate changes that are trauma related (PP versus $\mathrm{NC}$, and $\mathrm{CC}$ versus NC) and PTSD specific (PTSD versus CC). Although all subjects deactivated the mPFC and activated the insula for traumatic scripts, the PP deactivated the rostral anterior cingulate cortex (rACC) more than both control groups (CC and NC), but did not demonstrate ventromedial PFC (vmPFC) deactivation observed in controls. The findings observed only in the PTSD group (deactivation of the $\mathrm{ACC}$, and higher vMPFC activity) may reflect neural substrates specific to PTSD. ${ }^{37}$

Lanius and colleagues reported two fMRI studies where they used a script-driven symptom provocation paradigm. They also observed significantly decreased blood-oxygen-level dependent (BOLD) signal in the ventral ACC (Brodmann area 32) and the thalamus in the PTSD group to both the traumatic and nontraumatic emotional states conditions, suggesting that the earlier neuroimaging findings related to these areas in PTSD may not be specific to traumatic stimuli. ${ }^{38,39}$

There is also empirical evidence to suggest that reduced activation of the ACC may also be characteristic of pediatric PTSD. To date, the majority of studies have been performed in adult PTSD populations; only limited studies have been performed in children and/or adolescents with PTSD to investigate the neural correlates induced by symptom provocation. One small study examined brain responses during visual perception and imaginary recollection of traumatic reminders of adolescents (12 to 14 years) who developed PTSD versus those who did not after experiencing an earthquake. ${ }^{40}$ Sample size was limited (five with PTSD versus six trauma-exposed PTSDnegative individuals). During earthquake perception relative to neutral perception, the control group showed activation of the ACC, but the PTSD group did not. Additional analyses demonstrated that intergroup differences were significant, providing preliminary evidence that neurobiological alternation of the ACC in adolescence with PTSD are similar to those occurring in adult PTSD populations. ${ }^{40}$ 
Cognitive activation studies have also repeatedly demonstrated ACC to be implicated in the neurocircuitry associated with PTSD. Bremner et al used the modified Stroop task (color Stroop, emotional Stroop, and control task) and [15O] $\mathrm{H}_{2} \mathrm{O}$ PET to probe ACC function in 12 women with early CSA-related PTSD and nine CSA women without PTSD. The PTSD group demonstrated a relative decrease in ACC blood flow during the emotional but not the color Stroop task, which elicited increased $\mathrm{rCBF}$ in the ACC (BA 24 and 32 ) in both groups. ${ }^{41}$ Shin et al also investigated ACC functioning in 16 Vietnam combat veterans (eight with PTSD) using fMRI and an emotional counting Stroop paradigm. Subjects were asked to count the number of combat-related, generally negative and neutral words while being scanned. In the comparison of combat related to generally negative words, the nonPTSD group showed significant BOLD signal increases in rACC but the PTSD group did not. ${ }^{42}$ In addition, to assess interference processing and inhibitory control, tasks commonly associated with ACC function, a version of the counting Stroop task incorporating only effectively neutral words, also demonstrated hypoactivation of the ACC in patients with PTSD. This task requires participants to count the number of identical words presented on the screen, and to press a button corresponding to the correct number. In the interference condition, the presented word, a numerical value, is an "incorrect" response. This task was performed in 26 trauma exposed men, 13 of whom met diagnosis for PTSD. The PTSD group exhibited less deactivation in subgenual ACC and more deactivation in the insula as compared with controls during interference minus neutral task. ${ }^{43}$ Taken together, these data provide compelling evidence that functional impairments of the ACC and $\mathrm{mPFC}$ are associated with the neurocircuitry implicated in PTSD, and this holds for both symptom provocation and cognitive activations studies.

\section{Amygdala}

Another region implicated in the pathophysiology of PTSD is the amygdala. The amygdala is integral to the generation and maintenance of emotional responses, and this has been shown in both animal and human studies. ${ }^{44-46}$ In one of the first functional imaging symptom provocation studies, Rauch and colleagues ${ }^{47}$ used individualized trauma scripts and $[15 \mathrm{O}] \mathrm{H}_{2} \mathrm{O}$ PET in a small and heterogeneous group of eight PTSD subjects. They demonstrated increased regional cerebral blood flow $(\mathrm{rCBF})$ in anterior paralimbic (right posterior medialorbito frontal cortex [OFC], insular, anterior temporal polar, and medial temporal cortex) and limbic structures (amygdala) in the provoked versus control contrast group. In an ensuing study, our group exposed three groups of subjects (14 combat PTSD subjects, 11 combatexposed subjects without PTSD, and 11 combat unexposed healthy subjects) to combat sounds or white noise in two counterbalanced sessions and studied rCBF with $99 \mathrm{mTc}$ hexamethylpropyleneamineoxime (HMPAO) SPECT. Only the PTSD group showed increased $\mathrm{rCBF}$ in the left amygdaloid region..$^{48}$

Cognitive activation studies have also demonstrated the hyperresponsivity of the amygdala in PTSD. The amygdala is a region implicated in rapidly assessing the salience of emotional and especially threatrelated stimuli. ${ }^{49}$ A number of studies have presented fearful faces to individuals with PTSD using various exposure durations, and these have converged upon increased amygdala responsivity in PTSD (especially when stimulus presentation is rapid). ${ }^{50-52}$ Rauch et al compared amygdala responses in nine PTSD subjects versus eight combat-exposed, non-PTSD subjects using a previously validated masked emotional faces paradigm. Contrasting fearful versus happy masked faces revealed exaggerated amygdala responses in the PTSD subjects. Furthermore, the magnitude of these responses distinguished PTSD subjects with $75 \%$ sensitivity and $100 \%$ specificity. ${ }^{52}$
These findings suggest that PTSD is associated with increased amygdala responsivity to threat-related (but not necessarily trauma-related) stimuli. Another group used a similar masked emotional faces paradigm to examine 13 subjects with acute, rather than chronic PTSD. ${ }^{50}$ There was a positive correlation between the severity of PTSD and the difference in amygdala responses between masked fearful and happy faces. These findings suggest that functional abnormalities in brain responses to emotional stimuli observed in chronic PTSD might be apparent already in the acute phase. In a recent study, Bryant et al exposed 15 patients with PTSD and 15 age and sex-matched non-traumatized controls to fearful stimuli (16.7 ms) followed by a $163.3 \mathrm{~ms}$ neutral mask. They found significantly greater left amygdala activity in the PTSD relative to the control group. ${ }^{53}$

Regarding overt presentation of fearful faces, heightened amygdala activity does not appear to be particularly robust during this type of processing of fear stimuli, although differences have been obtained in some studies. ${ }^{51}$ In one study, Shin et al used overtly presented emotional facial expressions and fMRI to compare BOLD responses in 13 men with PTSD and 13 trauma-exposed men without PTSD. The PTSD group showed increased amygdala responses and decreased $\mathrm{mPFC}$ responses to overt fearful (versus happy) facial expressions. ${ }^{54}$ The amygdala is known to be involved with the rapid assessment of threat, and so it appears amydgala-related hyperactivation associated with PTSD is more sensitive to subliminal exposure durations in the region of 12-30 ms. Overt, longer duration exposure might lead to signal that integrates repeated stimulation and the habituation processes, and thus it might be less sensitive in picking up changes involving a specific process.

\section{Insula}

The insula is a large cortical structure composed of anatomical subdivisions that have distinct major afferent and efferent projec- 
tions; the anterior insula connects reciprocally with the amygdala, while the posterior insula connects reciprocally with the secondary primary sensory cortex and receives input from ventral posterior thalamic nuclei. The insula likely cannot be viewed as a single entity, as the anatomical subdivisions are thought to provide the basis for functional sub-regions. Meta-analysis of Wager and Barrett ${ }^{55}$ suggests that the ventral anterior insula is important for core affect and associated consciousness of subjective feelings. ${ }^{56}$ The dorsal anterior insula is thought to be involved in developing and updating motivational states, in autobiographical memory, ${ }^{57}$ cognitive control, elicit affective processing, and pain. ${ }^{55}$ In contrast, the posterior insula, including SII and portions of parietal operculum, are particularly specialized to convey homeostatic information such as pain, temperature, and sensual touch. ${ }^{58}$

Abnormal functioning of the insula has been linked to anxiety, with hyperactivation of the anterior insula demonstrated in highly anxious individuals when anticipating the arrival of aversive stimuli. ${ }^{59}$ Imaging studies specifically within PTSD populations have implicated the insula as one of the key regions involved in PTSD, particularly in regard to the anterior insula. For example, symptom provocation experiments have shown that during script-driven imagery with traumatic scripts (relative to neutral scripts), individuals with PTSD had higher anterior insula activation. ${ }^{60}$ This occurs in the region previously shown to be involved in interopceptive awareness of psychological and emotional states ${ }^{61}$ and autobiographical memory. ${ }^{57}$ Interestingly, a study investigating the influence of comorbid depression on the neurocorrelates of traumatic imagery found that individuals with PTSD but without comorbid depression had higher insula activation relative to a PTSD group with comorbid depression. ${ }^{62}$

\section{Functional Connectivity Analyses}

The introduction of functional connectivity studies reflects a growing awareness that complicated brain processes rely on the orchestrated interactions of distributed brain networks, rather than, or in addition to, activation of individual brain regions. Consequently functional connectivity analysis, which refers to the application of specific statistical methods to functional neuroimaging data sets to identify correlated brain activity across various regions, ${ }^{63,64}$ is a particularly relevant and useful technique. Several recent studies have applied functional connectivity analysis to neuroimaging studies of PTSD. Gilboa et al studied 20 individuals with a history of civilian trauma (10 with PTSD), using symptom provocation (autobiographical trauma-related and neutral scripts) and [15O] $\mathrm{H}_{2} \mathrm{O}$ PET. A multivariate analysis technique (partial least squares) was used to identify brain regions whose activity covaried with two reference ("seed") voxels, one in right PFC (BA 10) and the other in right amygdala. Amygdala activity was found to significantly influence activity in the visual cortex, subcallosal gyrus, and anterior cingulate in the PTSD subjects but not in the trauma-exposed controls. ${ }^{65}$ These findings indicate that blood flow measures reflect influence of the amygdala on medial frontal regions in PTSD, rather than a failure of mPFC inhibition of the amygdala. In addition, correlational analyses did not lend support for the failure of inhibition of the ACC over the amygdala.

Lanius et al used functional connectivity analyses on data gathered during fMRI script-driven symptom provocation experiments in 11 subjects with PTSD from sexual abuse/assault or motor vehicle accident (MVA), and 13 trauma-exposed subjects without PTSD. Connectivity maps for right ACC showed greater correlations in PTSD subjects (versus controls) in the right posterior cingulate cortex (PCC) (BA 29), right caudate, right parietal lobe (BA 7 and 40), and right occipital lobe (BA 19). Subjects without PTSD had greater correlations of ACC with left superior frontal gyrus (BA 9), left anterior ACC (BA 32), left striatum (caudate), left parietal lobe (BA 40 and 43), and left insula (BA 13). ${ }^{66}$ These findings are intriguing; however, our understanding of functional neural networks both in health and disease is still very limited. As methods for the analysis of functional connectivity continue to develop, and the knowledge base regarding coordinated activation of brain regions grows, these approaches will likely play an increasingly important role in delineating functional relationships between regions implicated in the pathophysiology of PTSD.

\section{Receptor Imaging}

Insight into which regions differ in activity as a function of PTSD can be gauged with fMRI, as BOLD signal is likely to reflect changes in neuronal firing or postsynaptic activity. In contrast, differences that may exist in functional neurochemistry between those with and without PTSD can be gauged using in vivo receptor imaging. Only a few studies to date have used receptor imaging to investigate neurotransmitter abnormalities associated with PTSD. Previous research in both animals and humans has implicated GABA, the principle inhibitory neurotransmitter within the brain, to be involved in both the pathogenesis and pathophysiology of PTSD. ${ }^{67,68}$ In a recent study, Geuze et al used [11C]flumazenil and PET to assess differences in the benzodiazepine-GABAA receptor complex in veterans with and without PTSD. ${ }^{69}$ Geuze et al found reduced binding potential of [11C]flumazenil in veterans with PTSD relative to control veterans without PTSD, specifically in the hippocampus, thalamus, and throughout the cortex, including the frontal, temporal, parietal, and occipital cortex. ${ }^{69}$ This suggests that these specific regions may be associated with pre-morbid differences in the composition/expression of GABAA-benzodiazepines receptors in PTSD patients, or a disease-induced modulation and/or downregulation of the GABAA receptor complex. Consistent with this study is a previous [123I]iomazenil SPECT study in Vietnam veterans, which also found decreased volume of distribu- 
tion of [123I]iomazenil in the prefrontal cortex in Vietnam veterans with PTSD relative to healthy controls. ${ }^{70}$ It should be noted, however, that another study also using [123I]iomazenil and SPECT was unable to find any differences in volume distribution of [123I]iomazenil between Gulf War veterans with and without PTSD. ${ }^{71}$

In a recent study, our group investigated altered mu-opioid receptor binding in PTSD using PET and the selective mu-opioid radiotracer [11C] carfentanil. $^{72}$ We had previously demonstrated endogenous opioids to be involved in inhibiting and modulating emotional responses in healthy humans, ${ }^{73}$ and this was the first study to provide direct evidence of alterations in mu-opioid receptor in vivo availability in PTSD. We demonstrated significant differences in the regional mu-opioid receptor BP2 between both the trauma-exposed groups and normal controls, as well as between PTSD patients and trauma exposed individuals who did not develop PTSD. These differences between the groups indicate mu-opioid receptor alterations arising from trauma can be differentiated from those specifically associated with PTSD. Changes were principally located in limbic forebrain and cortical regions known to be involved in emotion regulation, which likely reflect adaptive changes resulting from trauma exposure or stress, as well as maladaptive alterations associated with PTSD pathophysiology. ${ }^{72}$

Both receptor imaging and fMRI approaches have implicated similar brain regions in the pathophysiology of PTSD (eg, prefrontal cortex, amygdale, and hippocampus), yet reconciling the findings of these two methodologies poses a challenge. The receptor imaging data suggests decreased GABA binding potential in prefrontal regions in PTSD, suggesting potentially lower inhibitory tone, and therefore, enhanced reactivity. In contrast, the fMRI data indicates hypoactivation of cortical regions (specifically $\mathrm{mPFC}$ ) associated with PTSD. Alternatively, if the decreased GABA binding potential reflects a decreased number of GABAA receptors on the inhibitory interneurons, this could explain overall higher inhibitory tone in the mPFC. Furthermore, although BOLD activity likely reflects overall neuronal activity within a particular region, changes both in inhibitory and excitatory neurotransmission can contribute to the overall BOLD output. Thus, the changes in one particular neurotransmitter system should not be interpreted as defining the overall changes in BOLD signal. Moreover, given the complex interconnectivity of the PFC, its heterogeneity, and its broad functionality, it is possible that PTSD is associated with both increased and decreased activity within distinct regions of the PFC. To date, receptor imaging in PTSD has focused on inhibitory systems (GABA and opioids) and a focus for future research on glutamatergic (excitatory) systems, as well as other neurotransmitters/neuromodulators implicated in PTSD, such as serotonin, ${ }^{74}$ central catecholamines, ${ }^{75}$ and corticotrophin-releasing hormone ${ }^{76}$ will further help elucidate the molecular basis of altered brain function associated with PTSD.

\section{Treatment Studies and PTSD}

There is a growing appreciation that biomarkers that predict treatment response have the potential to significantly advance treatment of PTSD, and that neuroimaging is one of the key methodologies in search of those biomarkers. Moreover, insight into brain changes that occur after treatment can help elucidate the biological mechanisms involved. Consequently, treatment studies in PTSD patients that utilize neuroimaging are a rapidly growing area, although, only very limited evidence has been published so far. In one study, 14 treatment-seeking participants who were survivors of interpersonal violence $(n=9)$ or motor vehicle accidents $(n=5)$ received eight once-weekly sessions of CBT. ${ }^{77}$ Prior to treatment, participants viewed rapidly presented faces (fear and neutral) in a backwards masked paradigm. Increased bilateral amydala activity during fear processing prior to treatment was associated with poor response to CBT. Prior evidence suggests that adequate management of anxiety during therapy is required for CBT to be efficacious, despite CBT requiring activation of fear networks. ${ }^{78}$ Larger studies, using a variety of treatment strategies, can help reveal the generalizability and reliability of these findings.

\section{SUMMARY OF NEUROIMAGING STUDIES IN PTSD}

The studies reviewed above involve different cohorts (combat and CSA-related PTSD), different paradigms (symptom provocation versus cognitive activation), and different modalities (fMRI, PET, and SPECT) to assess structural, functional, and receptor alterations associated with PTSD. Despite these variations, the findings repeatedly converge on a number of key structures such as the amygdala, ACC, $\mathrm{mPFC}$, insula, and hippocampus. Taken together, the findings lend tentative support to a neurocircuitry model that emphasizes the role of dysregulation in threat-related processing in PTSD. According to this model, trauma exposure sets off a cascade of neural changes that culminates in a state of amygdala hyperresponsivity to trauma reminiscent and other threat-related stimuli that mediates symptoms of hyperarousal and vigilance associated with PTSD. The model also proposes associated inadequate top-down control by the mPFC and ACC that maintains and perpetuates the state of amygdala hyperresponsivity, and also helps mediate the failure to suppress attention to trauma-related stimuli. Consistent with this model, several studies have demonstrated reduced activation of the $\mathrm{mPFC}$ (BA 10 and 11) and ACC (BA 32) in PTSD subjects compared with traumatized controls. ${ }^{36,38,39,42,79}$ Other studies have reported increased responsivity of the amygdaloid region, ${ }^{45,47,52}$ although some have not. ${ }^{36,39,79}$ Although conceptualizing of PTSD-related pathophysiology as emphasizing the role of threat-related processing has some empirical support, there is clearly a need for a broader conceptualization of the processes implicated in the disorder. This is because deficits in threat-related processing explain only some aspects of PTSD, and 
other significant manifestations of PTSD remain unexplained by this model. These include emotional numbing, generalization of vigilance and avoidance from the initial traumatic event to other less closely related events, sensitization, and finally vulnerability and resilience factors.

To understand these complex phenomena, future neuroimaging research needs to focus on additional relevant mechanisms that may assist in understanding the complex phenomenology of PTSD such as fear conditioning and cognitive-emotion interactions. For example, behavioral and emotional avoidance associated with PTSD may be manifestations of underlying emotion dysregulation. The mPFC is a region known to be involved in emotion regulation, ${ }^{80}$ and evidence suggests that emotion regulation may be disrupted in PTSD. Posttraumatic stress symptoms have been associated with dysfunctional emotional processing strategies, such as impulse-control difficulties, limited access to effective emotion regulation strategies, and lack of emotional clarity. ${ }^{81}$

\section{REFERENCES}

1. Liberzon I, Phan KL. Brain-imaging studies of posttraumatic stress disorder. CNS Spectr. 2003;8(9):641-650.

2. Pitman RK, Shin LM, Rauch SL. Investigating the pathogenesis of posttraumatic stress disorder with neuroimaging. J Clin Psychiatry. 2001;62(Suppl 17):47-54.

3. Rauch SL, Shin LM. Functional neuroimaging studies in posttraumatic stress disorder. Ann NY Acad Sci. 1997;821:83-98.

4. McEwen BS, Sapolsky RM. Stress and cognitive function. Curr Opin Neurobiol. 1995;5(2):205-216.

5. O'Brien JT, Ames D, Schweitzer I, Colman P, Desmond P, Tress B. Clinical and magnetic resonance imaging correlates of hypothalamic-pituitary-adrenal axis function in depression and Alzheimer's disease. Br J Psychiatry. 1996;168(6):679-687.

6. McEwen BS. Possible mechanisms for atrophy of the human hippocampus. Mol Psychiatry. 1997;2(3):255-262.

7. Corcoran KA, Maren S. Hippocampal inactivation disrupts contextual retrieval of fear memory after extinction. J Neurosci. 2001;21(5):1720-1726.

8. Eichenbaum H. A cortical-hippocampal system for declarative memory. Nat Rev Neurosci. 2000;1(1):41-50.
9. Bremner JD, Randall P, Scott TM, et al. MRI-based measurement of hippocampal volume in patients with combat-related posttraumatic stress disorder. Am J Psychiatry. 1995;152(7):973-981.

10. Bremner JD, Scott TM, Delaney RC, et al. Deficits in short-term memory in posttraumatic stress disorder. Am J Psychiatry. 1993;150(7):1015-1019.

11. McNally RJ, Lasko NB, Macklin ML, Pitman RK. Autobiographical memory disturbance in combat-related posttraumatic stress disorder. $\mathrm{Be}$ hav Res Ther. 1995;33(6):619-630.

12. Bremner JD, Randall P, Vermetten E, et al. Magnetic resonance imaging-based measurement of hippocampal volume in posttraumatic stress disorder related to childhood physical and sexual abuse - a preliminary report. Biol Psychiatry. 1997;41(1):23-32.

13. Gurvits TV, Shenton ME, Hokama H, et al. Magnetic resonance imaging study of hippocampal volume in chronic, combat-related posttraumatic stress disorder. Biol Psychiatry. 1996;40(11):1091-1099.

14. Stein MB, Koverola C, Hanna C, Torchia MG, McClarty B. Hippocampal volume in women victimized by childhood sexual abuse. Psychol Med. 1997;27(4):951-959.

15. Villarreal G, Hamilton DA, Petropoulos H, et al. Reduced hippocampal volume and total white matter volume in posttraumatic stress disorder. Biol Psychiatry. 2002;52(2):119-125.

16. Schuff N, Neylan TC, Lenoci MA, et al. Decreased hippocampal $\mathrm{N}$-acetylaspartate in the absence of atrophy in posttraumatic stress disorder. Biol Psychiatry. 2001;50(12):952-959.

17. Villarreal G, Petropoulos H, Hamilton DA, et al. Proton magnetic resonance spectroscopy of the hippocampus and occipital white matter in PTSD: preliminary results. Can J Psychiatry. 2002;47(7):666-670.

18. Shin LM, Shin PS, Heckers S, et al. Hippocampal function in posttraumatic stress disorder. Hippocampus. 2004;14(3):292-300.

19. Bonne O, Brandes D, Gilboa A, et al. Longitudinal MRI study of hippocampal volume in trauma survivors with PTSD. Am J Psychiatry. 2001;158(8):1248-1251.

20. Carrion VG, Weems CF, Eliez S, et al. Attenuation of frontal asymmetry in pediatric posttraumatic stress disorder. Biol Psychiatry. 2001;50(12):943-951.

21. De Bellis MD, Keshavan MS, Clark DB, et al. A.E. Bennett Research Award. Developmental traumatology. Part II: Brain development. Biol Psychiatry. 1999;45(10):1271-1284.

22. Karl A, Schaefer M, Malta LS, Dörfel D, Rohleder N, Werner A. A meta-analysis of structural brain abnormalities in PTSD. Neurosci Biobehav Rev. 2006;30(7):1004-1031.

23. Gilbertson MW, Shenton ME, Ciszewski A, et al. Smaller hippocampal volume predicts pathologic vulnerability to psychological trauma. Nat Neurosci. 2002;5(11):1242-1247.

24. Gilbertson MW, Williston SK, Paulus LA, et al.
Configural cue performance in identical twins discordant for posttraumatic stress disorder: theoretical implications for the role of hippocampal function. Biol Psychiatry. 2007;62(5):513-520.

25. Woodward SH, Kaloupek DG, Streeter CC, Martinez C, Schaer M, Eliez S. Decreased anterior cingulate volume in combat-related PTSD. Biol Psychiatry. 2006;59(7):582-587.

26. Corbo V, Clement MH, Armony JL, Pruessner JC, Brunet A. Size versus shape differences: contrasting voxel-based and volumetric analyses of the anterior cingulate cortex in individuals with acute posttraumatic stress disorder. Biol Psychiatry. 2005;58(2):119-124.

27. Kasai K, Yamasue H, Gilbertson MW, Shenton ME, Rauch SL, Pitman RK. Evidence for acquired pregenual anterior cingulate gray matter loss from a twin study of combat-related posttraumatic stress disorder. Biol Psychiatry. 2008;63(6):550-556.

28. Fennema-Notestine C, Stein MB, Kennedy CM, Archibald SL, Jernigan TL. Brain morphometry in female victims of intimate partner violence with and without posttraumatic stress disorder. Biol Psychiatry. 2002;52(11):1089-1101.

29. Woon FL, Hedges DW. Hippocampal and amygdala volumes in children and adults with childhood maltreatment-related posttraumatic stress disorder: a meta-analysis. Hippocampus. 2008;18(8):729-736.

30. Augustine JR. Circuitry and functional aspects of the insular lobe in primates including humans. Brain Res Brain Res Rev. 1996;22(3):229-244.

31. Phillips ML, Drevets WC, Rauch SL, Lane R. Neurobiology of emotion perception I: The neural basis of normal emotion perception. Biol Psychiatry. 2003;54(5):504-514.

32. Chen S, Xia W, Li L, et al. Gray matter density reduction in the insula in fire survivors with posttraumatic stress disorder: a voxelbased morphometric study. Psychiatry Res. 2006;146(1):65-72.

33. Bush G, Luu P, Posner MI. Cognitive and emotional influences in anterior cingulate cortex. Trends Cogn Sci. 2000;4(6):215-222.

34. Shin LM, Kosslyn SM, McNally RJ, et al. Visual imagery and perception in posttraumatic stress disorder. A positron emission tomographic investigation. Arch Gen Psychiatry. 1997;54(3):233-241.

35. Bremner JD, Narayan M, Staib LH, Southwick SM, McGlashan T, Charney DS. Neural correlates of memories of childhood sexual abuse in women with and without posttraumatic stress disorder. Am J Psychiatry. 1999;156(11):1787-1795.

36. Shin LM, McNally RJ, Kosslyn SM, et al. Regional cerebral blood flow during script-driven imagery in childhood sexual abuse-related PTSD: a PET investigation. Am J Psychiatry. 1999;156(4):575-584.

37. Britton JC, Phan KL, Taylor SF, Fig LM, Liberzon I. Corticolimbic blood flow in posttraumatic stress disorder during script-driven imagery. Biol Psychiatry. 2005;57(8):832-840. 
38. Lanius RA, Hopper JW, Menon RS. Individual differences in a husband and wife who developed PTSD after a motor vehicle accident: a functional MRI case study. Am J Psychiatry. 2003;160(4):667-669.

39. Lanius RA, Williamson PC, Densmore M, et al. Neural correlates of traumatic memories in posttraumatic stress disorder: a functional MRI investigation. Am J Psychiatry. 2001;158(11):1920-1922.

40. Hsu CC, Chong MY, Yang P, Yen CF. Posttraumatic stress disorder among adolescent earthquake victims in Taiwan. J Am Acad Child Adolesc Psychiatry. 2002;41(7):875-881.

41. Bremner JD, Vermetten E, Vythilingam M, et al. Neural correlates of the classic color and emotional stroop in women with abuse-related posttraumatic stress disorder. Biol Psychiatry. 2004;55(6):612-620.

42. Shin LM, Whalen PJ, Pitman RK, et al. An fMRI study of anterior cingulate function in posttraumatic stress disorder. Biol Psychiatry. 2001;50(12):932-942.

43. Shin LM, Bush G, Whalen PJ, et al. Dorsal anterior cingulate function in posttraumatic stress disorder. J Trauma Stress. 2007;20(5):701-712.

44. Davis M. The role of the amygdala in fear and anxiety. Annu Rev Neurosci. 1992;15:353-375.

45. Liberzon I, Taylor SF, Amdur R, et al. Brain activation in PTSD in response to trauma-related stimuli. Biol Psychiatry. 1999;45(7):817-826

46. Morgan MA, LeDoux JE. Differential contribution of dorsal and ventral medial prefrontal cortex to the acquisition and extinction of conditioned fear in rats. Behav Neurosci. 1995;109(4):681-688.

47. Rauch SL, van der Kolk BA, Fisler RE, et al. A symptom provocation study of posttraumatic stress disorder using positron emission tomography and script-driven imagery. Arch Gen Psychiatry. 1996;53(5):380-387.

48. Liberzon I, Britton JC, Phan KL. Neural correlates of traumatic recall in posttraumatic stress disorder. Stress. 2003;6(3):151-156.

49. Davis M, Whalen PJ. The amygdala: vigilance and emotion. Mol Psychiatry. 2001;6(1):13-34.

50. Armony JL, Corbo V, Clement MH, Brunet A. Amygdala response in patients with acute PTSD to masked and unmasked emotional facial expressions. Am J Psychiatry. 2005;162(10):1961-1963.

51. Bryant RA, Kemp AH, Felmingham KL, et al. Enhanced amygdala and medial prefrontal activation during nonconscious processing of fear in posttraumatic stress disorder: an fMRI study. Hum Brain Mapp. 2008;29(5):517-523.

52. Rauch SL, Whalen PJ, Shin LM, et al. Exaggerated amygdala response to masked facial stimuli in posttraumatic stress disorder: a functional MRI study. Biol Psychiatry. 2000;47(9):769-776.

53. Bryant RA, Kemp AH, Felmingham KL, et al. Enhanced amygdala and medial prefrontal activation during nonconscious processing of fear in posttraumatic stress disorder: sn fMRI study. Hum Brain Mapp. 2008;29(5):517-523.
54. Shin LM, Wright CI, Cannistraro PA, et al. A functional magnetic resonance imaging study of amygdala and medial prefrontal cortex responses to overtly presented fearful faces in posttraumatic stress disorder. Arch Gen Psychiatry. 2005;62(3):273-281.

55. Wager TD, Feldman Barrett L. From affect to control: functionalspecialization of the insula in motivation and regulation. http://www.columbia.edu/cu/psychology/tor/Papers/Wager_Feldman_Barrett_2004_Insula_meta-analysis.pdf. Accessed May 28, 2009.

56. Craig AD. How do you feel? Interoception: the sense of the physiological condition of the body. Nat Rev Neurosci. 2002;3(8):655-666.

57. Fink GR, Markowitsch HJ, Reinkemeier M, Bruckbauer T, Kessler J, Heiss WD. Cerebral representation of one's own past: neural networks involved in autobiographical memory. $J$ Neurosci. 1996;16(13):4275-4282.

58. Rolls ET, Grabenhorst F, Parris BA. Warm pleasant feelings in the brain. Neuroimage. 2008;41(4):1504-1513

59. Simmons A, Strigo I, Matthews SC, Paulus MP, Stein MB. Anticipation of aversive visual stimuli is associated with increased insula activation in anxiety-prone subjects. Biol Psychiatry. 2006;60(4):402-409.

60. Hopper JW, Frewen PA, van der Kolk BA, Lanius RA. Neural correlates of reexperiencing, avoidance, and dissociation in PTSD: symptom dimensions and emotion dysregulation in responses to script-driven trauma imagery. $J$ Trauma Stress. 2007;20(5):713-725.

61. Critchley HD, Wiens S, Rotshtein P, Ohman A, Dolan RJ. Neural systems supporting interoceptive awareness. Nat Neurosci. 2004;7(2):189-195.

62. Lanius RA, Frewen PA, Girotti M, Neufeld RW, Stevens TK, Densmore M. Neural correlates of trauma script-imagery in posttraumatic stress disorder with and without comorbid major depression: a functional MRI investigation. Psychiatry Res. 2007;155(1):45-56.

63. Friston KJ, Frith CD, Fletcher P, Liddle PF, Frackowiak RS. Functional topography: multidimensional scaling and functional connectivity in the brain. Cereb Cortex. 1996;6(2):156-164.

64. Friston KJ, Frith CD, Liddle PF, Frackowiak RS. Functional connectivity: the principal-component analysis of large (PET) data sets. J Cereb Blood Flow Metab. 1993;13(1):5-14.

65. Gilboa A, Shalev AY, Laor L, et al. Functional connectivity of the prefrontal cortex and the amygdala in posttraumatic stress disorder. Biol Psychiatry. 2004;55(3):263-272.

66. Lanius RA, Williamson PC, Densmore M, et al. The nature of traumatic memories: a 4-T FMRI functional connectivity analysis. Am J Psychiatry. 2004;161(1):36-44.

67. Harvey BH, Oosthuizen F, Brand L, Wegener G, Stein DJ. Stress-restress evokes sustained iNOS activity and altered GABA levels and NMDA receptors in rat hippocampus. Psychopharmacology (Berl). 2004;175(4):494-502.
68. Vaiva G, Thomas P, Ducrocq F, et al. Low posttrauma GABA plasma levels as a predictive factor in the development of acute posttraumatic stress disorder. Biol Psychiatry. 2004;55(3):250-254.

69. Geuze E, van Berckel BNM, Lammertsma AA, et al. Reduced GABAA benzodiazepine receptor binding in veterans with posttraumatic stress disorder. Mol Psychiatry. 2008;13(1):74-83.

70. Bremner JD, Innis RB, Southwick SM, Staib L Zoghbi SS, Charney DS. Decreased benzodiazepine receptor binding in prefrontal cortex in combat-related posttraumatic stress disorder. Am J Psychiatry. 2000;157(7):1120-1126.

71. Fujita M, Southwick SM, Denucci CC, et al Central type benzodiazepine receptors in Gulf War veterans with posttraumatic stress disorder. Biol Psychiatry. 2004;56(2):95-100).

72. Liberzon I, King AP, Britton JC, Phan KL, Abelson JL, Taylor SF. Paralimbic and medial prefrontal cortical involvement in neuroendocrine responses to traumatic stimuli. Am J Psychiatry. 2007;164(8):1250-1258.

73. Phan KL, Wager T, Taylor SF, Liberzon I. Functional neuroanatomy of emotion: a metaanalysis of emotion activation studies in PET and fMRI. Neuroimage. 2002;16(2):331-348.

74. Southwick SM, Krystal JH, Bremner JD, et al. Noradrenergic and serotonergic function in posttraumatic stress disorder. Arch Gen Psychiatry. 1997;54(8):749-758.

75. Yehuda R, Southwick SM, Giller EL, Ma XW, Mason JW. Urinary catecholamine excretion adn severity of PTSD symptoms in vietnam combat veterans. J Nerv Ment Dis. 1992;180(5):321-325.

76. Bremner JD, Licinio J, Darnell A, et al. Elevated CSF corticotropin-releasing factor concentrations in posttraumatic stress disorder. Am J Psychiatry. 1997;154(5):624-629.

77. Bryant RA, Felmingham K, Kemp A, et al. Amygdala and ventral anterior cingulate activation predicts treatment response to cognitive behaviour therapy for posttraumatic stress disorder. Psychol Med. 2008;38(4):555-561.

78. Foa EB, Meadows EA. Psychosocial treatments for posttraumatic stress disorder: a critical review. Annu Rev Psychol. 1997;48:449-480.

79. Bremner JD, Staib LH, Kaloupek D, Southwick SM, Soufer R, Charney DS. Neural correlates of exposure to traumatic pictures and sound in Vietnam combat veterans with and without posttraumatic stress disorder: a positron emission tomography study. Biol Psychiatry. 1999;45(7):806-816.

80. Ochsner KN, Bunge SA, Gross JJ, Gabrieli JD. Rethinking feelings: an FMRI study of the cognitive regulation of emotion. $J$ Cogn Neurosci. 2002;14(8):1215-1229.

81. Tull MT, Barrett HM, McMillan ES, Roemer L. A preliminary investigation of the relationship between emotion regulation difficulties and posttraumatic stress symptoms. Behav Ther. 2007;38(3):303-313. 